\title{
Author Correction: Znf179 induces differentiation and growth arrest of human primary glioblastoma multiforme in a p53-dependent cell cycle pathway
}

\author{
Kuen-Haur Lee ${ }^{1}$, Chi-Long Chen ${ }^{2,3}$, Yi-Chao Lee ${ }^{4}$, Tzu-Jen Kao ${ }^{4}$, Kai-Yun Chen ${ }^{4}$, \\ Chih-Yeu Fang ${ }^{5}$, Wen-Chang Chang ${ }^{6}$, Yung-Hsaio Chiang ${ }^{4} \&$ Chi-Chen Huang $\mathbb{D}^{4}$ \\ Correction to: Scientific Reports https://doi.org/10.1038/s41598-017-05305-0, published online 06 July 2017
}

In the original version of this Article, Chi-Long Chen and Chih-Yeu Fang were incorrectly listed as being affiliated with "Pathology Department, Taipei Medical University, Taipei, Taiwan". The correct affiliations are listed below:

\section{Chi-Long Chen}

Department of Pathology, Taipei Medical University Hospital, Taipei, Taiwan

Department of Pathology, School of Medicine, College of Medicine, Taipei Medical University, Taipei, Taiwan

Chih-Yeu Fang

Department of Pathology, Wan Fang Hospital, Taipei Medical University, Taipei, Taiwan

This error has now been corrected in the PDF and HTML versions of this Article, and in the accompanying Supplementary figures and legends file.

(c) (i) Open Access This article is licensed under a Creative Commons Attribution 4.0 International cc. License, which permits use, sharing, adaptation, distribution and reproduction in any medium or format, as long as you give appropriate credit to the original author(s) and the source, provide a link to the Creative Commons license, and indicate if changes were made. The images or other third party material in this article are included in the article's Creative Commons license, unless indicated otherwise in a credit line to the material. If material is not included in the article's Creative Commons license and your intended use is not permitted by statutory regulation or exceeds the permitted use, you will need to obtain permission directly from the copyright holder. To view a copy of this license, visit http://creativecommons.org/licenses/by/4.0/.

(c) The Author(s) 2018

${ }^{1}$ Graduate Institute of Cancer Biology and Drug Discovery, College of Medical Science and Technology, Taipei Medical University, Taipei, Taiwan. ${ }^{2}$ Department of Pathology, Taipei Medical University Hospital, Taipei, Taiwan. ${ }^{3}$ Department of Pathology, School of Medicine, College of Medicine, Taipei Medical University, Taipei, Taiwan. ${ }^{4}$ Graduate Institute of Neural Regenerative Medicine, College of Medical Science and Technology, Taipei Medical University, Taipei, Taiwan. ${ }^{5}$ Department of Pathology, Wan Fang Hospital, Taipei Medical University, Taipei, Taiwan. ${ }^{6}$ Graduate Institute of Medical Sciences, College of Medicine, Taipei Medical University, Taipei, Taiwan. Kuen-Haur Lee and Chi-Long Chen contributed equally. Correspondence and requests for materials should be addressed to C.-C.H. (email: hcc0609@tmu.edu.tw) 